\title{
TMEFF2 is an endogenous inhibitor of the CRH signal transduction pathway
}

\author{
Marta Labeur, Barbara Wölfel, Johanna Stalla and Günter K Stalla
}

Department of Neuroendocrinology, Max Planck Institute of Psychiatry, 80804 Munich, Germany

Correspondence should be addressed to M Labeur; G K Stalla Emails

labeur@mpipsykl.mpg.de; stalla@mpipsykl.mpg.de

\begin{abstract}
TMEFF2 is a transmembrane protein with unknown function, containing an altered epidermal growth factor (EGF)-like motif, two follistatin-like domains, and a cytosolic tail with a putative G-protein-activating motif. TMEFF2 is predominantly expressed in brain and prostate and has been implicated in cell signaling, neuronal cell survival, and tumor suppression.

We found that expression of TMEFF2 in pituitary corticotrope cells inhibits the effects of corticotropin-releasing hormone $(\mathrm{CRH})$ on the production of intracellular CAMP, and CREB, and transcription of Pomc. Regulation of the activity of CRH by TMEFF2 requires neither the cytoplasmic tail nor the EGF domain, while deletion of the follistatin modules abolishes the inhibitory function of TMEFF2. Moreover, a soluble secreted protein containing the complete extracellular domain is sufficient for inhibition of $\mathrm{CRH}$ signaling. TMEFF2-induced inhibition depends on serum components. Furthermore, TMEFF2 regulates the non-canonical activin/BMP4 signaling, PI3K, and Ras/ERK1/2 pathways. Thus, TMEFF2 inhibits the CRH signaling pathway and the PI3K/AKT and Ras/ERK1/2 pathways, contributing to a significant inhibition of transcription of Pomc. We found that expression of TMEFF2 in human Cushing's adenoma is reduced when compared with normal human pituitary, which may indicate that TMEFF2 acts as a tumor suppressor in these adenomas. Furthermore, the overexpression of TMEFF2 decreased proliferation of corticotrope cells. Our results indicate a potential therapeutic use of TMEFF2 or factors that stimulate the activity of TMEFF2 for the treatment of corticotrope tumors in order to reduce their secretion of ACTH and proliferation.
\end{abstract}
Key Words
- TMEFF2
- $\mathrm{CRH}$
- Cushing's
- POMC

Journal of Molecular Endocrinology (2015) 54, 51-63

\section{Introduction}

Corticotropin-releasing hormone $(\mathrm{CRH})$ plays a central role in the regulation of the hypothalamic-pituitaryadrenal axis, mediating endocrine and behavioral responses to various stressors (Perrin \& Vale 1999, Grammatopoulos \& Chrousos 2002, Reul \& Holsboer 2002, Bale \& Vale 2004). At the pituitary level, CRH stimulates the expression of pro-opiomelanocortin (Pomc) and production of adrenocorticotropin (ACTH) (Gagner \&
Drouin 1987) by the G protein-coupled receptor (GPCR), CRH receptor 1 (CRHR1). CRH results in Gs-mediated stimulation of adenylate cyclase (AC), leading to increased levels of intracellular cAMP. cAMP activates protein kinase A (PKA), a tetrameric holoenzyme consisting of two catalytic and two regulatory units. Upon binding of cAMP to the regulatory units, the activated catalytic units phosphorylate different transcription factors, which in 
turn activate the Pomc promoter. cAMP activates calcium/ calmodulin kinase II and Rap1/B-raf/ERK1/2 (Kovalovsky et al. 2002) to induce expression of Nur77/Nurr1 (Murphy \& Conneely 1997, Philips et al. 1997, Maira et al. 1999), which then triggers transcription of Pomc via the NurRE consensus site in the promoter of Pomc. CRH also induces the transcriptional activity of activating protein 1 (AP1) and CREB, which promotes transcription of Pomc through the AP1-binding site located in the first exon (Boutillier et al. 1995, 1998).

Besides CRH, other hypothalamic hormones including arginine vasopressin (AVP) stimulate expression of Pomc in pituitary corticotropes (Tse \& Lee 1998, Birnbaumer 2000). Other pituitary paracrine factors such as cytokines of the gp130 family also influence transcription of Pomc (Chesnokova \& Melmed 2002).

Overproduction of ACTH by pituitary adenomas leads to excessive secretion of glucocorticoids from the adrenal glands, which causes endogenous Cushing's syndrome (Orth 1995, Dahia \& Grossman 1999). The underlying mechanisms responsible for secretion of ACTH from pituitary corticotropinomas remain poorly understood. As a consequence, no effective drug therapy is currently available, particularly if surgical excision is not successful.

We have previously demonstrated that retinoic acid (RA) inhibits CRH/CRHR1 signaling in corticotrope cells (Paez-Pereda et al. 2001) and induces BMP4, which participates in the antiproliferative effects of RA (Giacomini et al. 2006). Moreover, treatment of dogs with ACTHsecreting tumors with RA controls hypersecretion of ACTH and cortisol, leading to resolution of the clinical symptoms (Castillo et al. 2006). Long-term treatment with RA proved to be beneficial in patients with Cushing's disease (Pecori Giraldi et al. 2012). In order to elucidate the molecular events that mediate the effects of RA in corticotrope cells, we applied, in preliminary experiments, cDNA microarray technology (M Labeur \& G K Stalla, unpublished observations). Biostatistic evaluation of acquired data identified several regulated candidates, from which the transmembrane protein containing an altered epidermal growth factor (EGF)-like motif and two follistatinlike domains, TMEFF2, was chosen for further investigation.

TMEFF1 and TMEFF2 are novel members of the EGF-like protein family and are structurally unique within this family (Kanemoto et al. 2001). TMEFF2, also called tomoregulin 2, TPEF, TENB2, and HPP, is a transmembrane protein with unknown function. It has been deduced to encode a 368 amino acid protein, which contains a predicted N-terminal signal peptide, extracellular domains consisting of an altered EGF-like repeat and two follistatin modules, and a transmembrane domain connected to a cytosolic tail with a putative G-proteinactivating motif. TMEFF2 is predominantly expressed in prostate and brain (Uchida et al. 1999, Horie et al. 2000, Liang et al. 2000, Glynne-Jones et al. 2001, Young et al. 2001, Gery et al. 2002) and has been implicated in cell signaling (Uchida et al. 1999), neuronal cell survival (Horie et al. 2000), and tumor suppression (Liang et al. 2000, Young et al. 2001, Gery et al. 2002).

Although the function of TMEFF2 is almost unknown, its structural domains indicate that it may have a role in the regulation of growth factor signaling either as a ligand precursor, a membrane-bound receptor with a putative $\mathrm{G}$ protein-binding domain, or as a binding protein for growth factors. As there is no previous evidence of an involvement of TMEFF2 in CRH signaling and as RA, a CRH signaling pathway inhibitor, may modulate TMEFF2, we decided to investigate in detail the effects of TMEFF2 on the signal transduction pathway of $\mathrm{CRH}$.

\section{Materials and methods}

\section{Cell culture and chemicals}

AtT20 pituitary corticotrope tumor cells (Leung et al. 1982) and GH3 pituitary lactosomatotrope tumor cells (American Type Culture Collection, Manassas, VA, USA) were cultured in DMEM supplemented with 10\% FCS, $2 \mathrm{mM}$ glutamine, $10^{5} \mathrm{U} / 1$ penicillin and streptomycin, and $2.5 \mathrm{mg} / 1$ amphotericin $\mathrm{B}$.

Recombinant mouse BMP4, mouse/rat/human activin A, and interleukin 6 (IL6) were purchased from R\&D Systems (Minneapolis, MN, USA). CRH and AVP were obtained from Bachem (Heidelberg, BW, Germany). Forskolin and CPT-cAMP were purchased from Sigma Chemical Co.

\section{Human tissues}

Experiments were carried out according to the guidelines of the Ethical Committee of the Max Planck Institute for using autopsy and biopsy materials. Human pituitary tissues were obtained from autopsies performed 8-12 h after postmortem in healthy subjects after sudden accidental death. Human pituitary adenomas were obtained after their surgical resection from patients with Cushing's disease. Tissue fragments from both normal pituitaries and pituitary adenomas were shock frozen on dry ice and stored at $-80^{\circ} \mathrm{C}$.

Published by Bioscientifica Ltd 


\section{RT-PCR}

Total cellular RNA was isolated from excised tissues (human pituitary gland and human pituitary adenomas) using TRIzol reagent (Invitrogen). RNA $(1 \mu \mathrm{g})$ was used for cDNA synthesis with Superscript II reverse transcriptase (Invitrogen) according to the manufacturer's instructions. The following primers were used: TMEFF2 sense primer 5'-CTGATGGGAAATCTTATGATAATG-3' and antisense primer 5'-CAGGAACAACGTAGAGAACACTGT-3'. Internal control was performed by amplifying $\beta$-ACTIN from the same human samples (human $\beta$-ACTIN sense primer: $5^{\prime}$-ACGGGGTCACCCACACTGTGC-3' and antisense primer: $5^{\prime}$-CTAGAAGCATTTGCGGTGGACGATG-3'). Band intensity of TMEFF2 was normalized with $\beta$-ACTIN in the same samples.

\section{Immunohistochemistry}

All tissues were fixed in 4\% phosphate-buffered paraformaldehyde (Sigma). Sections measuring $8 \mu \mathrm{m}$ in diameter were immunostained with an anti-human TMEFF2 antibody (R\&D Systems) in combination with a biotinylated secondary antibody (Vector Laboratories, Burlingame, CA, USA) using the avidin-biotin-peroxidase method. Immunoreactivity was visualized with diaminobenzidine (Sigma) and $0.01 \%$ hydrogen peroxide and then counterstained with toluidine blue.

ACTH was detected with a MAB (Dako Diagnostika, Hamburg, Germany) in combination with an anti-mouse IgG (Sigma) and mouse alkaline phosphatase (AP)-anti AP complex (Sigma) with Vector Red (Vector Laboratories), according to the manufacturer's instructions. Negative controls were performed without the primary antibody.

\section{Plasmids and transfection assays}

The Pomc-Luc plasmid containing the luciferase gene under the control of $770 \mathrm{bp}$ of the rat Pomc promoter includes all the necessary sequences for the expression and regulation of Pomc (Therrien \& Drouin 1991, Liu et al. 1995).

The NurRE-Luc construct containing three copies of the NurRE coupled to the minimal Pomc promoter $(-34 /+63)$ were provided by Dr J Drouin, Laboratoire de Génétique Moléculaire, Institut de Recherches Cliniques de Montréal, Québec, Canada. The CRE-Luc construct (Clontech Laboratories, Inc.) contains the cAMP-responsive element upstream of the TATA box of the herpes simplex virus thymidine kinase promoter and the reporter gene luciferase. The Gal4-Elk1 expression vector was provided by Dr Phillip Stork (Vossler et al. 1997) and the pG5-Luc construct was obtained from Promega Corp. An expression vector coding for the PKA catalytic subunit was used (Maurer 1989). The TMEFF2 expression vector and mutated plasmids were provided by Affectis Pharmaceutical AG, (Munich, BY, Germany). TMEFF2 coding sequence and its mutants were inserted either into the pcDNA-DEST40Gateway Vector (Invitrogen) or into the mammalian expression vector pcDNA3.1 (Invitrogen).

Cell transfections were performed using either Lipofectamine or Lipofectamine 2000 (Invitrogen) according to the manufacturer's instructions. RSV- $\beta$-galactosidase was used as an internal control. After $18 \mathrm{~h}$ in culture medium, cells were incubated with the indicated compounds (100 nM CRH, $10 \mu \mathrm{M}$ forskolin, $20 \mathrm{ng} / \mathrm{ml}$ activin, $100 \mathrm{ng} / \mathrm{ml} \mathrm{BMP} 4$, and $50 \mu \mathrm{M}$ CPT-cAMP). At the end of the treatment, the protein lysate was collected and luciferase and $\beta$-galactosidase activities were measured using the Berthold Tristar luminometer and the Dynatech MR5000 microplate reader respectively.

\section{AtT20 stable clones}

AtT20 cells were stably transfected with a TMEFF2 expression vector or the corresponding control vector. Cells were provided by Affectis Pharmaceutical AG.

\section{SIRNA}

The sequences of the two mouse Tmeff 2 siRNA oligonucelotides used were 5'-UCAGAAGGAUCCUGUGCUA-3' and 5'-CGGUUACGAUGACAGAGAA-3'. Non-specific control RNA (control siRNA) oligonucleotides with similar GC content compared with siRNA were used as control (MWG-Biotech, Ebersberg, Germany). Cell transfection was performed using Lipofectamine (Invitrogen) in Opti-MEM medium with $10 \mu \mathrm{l}$ Lipofectamine and $50 \mathrm{nM}$ Tmeff 2 siRNA or control siRNA per well. After $6 \mathrm{~h}$, the transfection medium was removed and the cells were cultured in DMEM supplemented with $2 \%$ FCS. Cells were stimulated as indicated.

\section{Cell proliferation and colony formation assay in soft agar}

After $18 \mathrm{~h}$, transfected cells were incubated in DMEM medium containing 10\% FCS. The following day a WST-1 assay (Roche Molecular Biochemicals) was used to assess cell proliferation and cell viability following the manufacturer's instructions (Paez-Pereda et al. 2000). The values of the background absorbance were subtracted from the absorbances of all samples. Acridine orange-ethidium bromide staining was used to rule out toxic effects.

Published by Bioscientifica Ltd. 
Anchorage-independent colony formation was determined using AtT20 cells transfected with a TMEFF2 expression vector as follows. After $24 \mathrm{~h}$, transfected cells were suspended in $0.3 \%$ agar with complete DMEM medium and plated in $85-\mathrm{mm}$ dishes previously coated with $0.5 \%$ agar. The cultures were maintained at $37^{\circ} \mathrm{C}$ for 20 days.

\section{Hormone measurements}

AtT20 cells (20 000) stably transfected with a TMEFF2 expression vector or the empty vector were plated in a 96-well plate. After plating for $24 \mathrm{~h}$, the cells were incubated in DMEM with $10 \%$ FCS for 6,18 , and $24 \mathrm{~h}$. Supernatants were stored at $-80^{\circ} \mathrm{C}$ until measurement of ACTH was performed. ACTH was measured by RIA as described previously (Paez-Pereda. et al. 2000).

\section{Western blotting}

AtT20 cells were transfected with a TMEFF2 expression vector or the empty vector and treated as indicated. In other sets of experiments, AtT20 cells were transfected with TMEFF 2 siRNA or with control siRNA as indicated above. Cell lysates were prepared by resuspending the cells in RIPA buffer and analyzed by western blotting. Equal levels of protein were resolved on 10\% Tris/Glycin gel (Anamed Electrophorese Gmbh, Gross-Bieberau, Germany), transferred onto PVDF membrane and immunoblotted with anti-TMEFF2 antibody (R\&D Systems, Wiesbaden, Germany), anti-phospho-p44/42 MAPK (ERK1/2) (Thr202/Tyr204) antibody, or anti-phospho-AKT (Ser473) antibody. Immunodetection was possible with the use of HRP-conjugated rabbit antibody, followed by incubation with ECL/ECL Plus detection reagents (Amersham Pharmacia Biotech). After stripping, the membranes were treated with either anti-total ERK1/2 antibody, anti-total AKT antibody (all from Cell Signaling, Danvers, MA, USA), or anti- $\beta$-actin antibody (Chemicon, Temecula, CA, USA).

\section{CAMP RIA}

Radioimmunological determination of cAMP was performed with a commercial RIA kit from NEN Life Science Products, Inc. (Boston, MA, USA). In brief, $24 \mathrm{~h}$ after transfection, AtT20 cells were cultured in 48-well plates. The following day, cells were washed and stimulated for $1 \mathrm{~h}$ with $100 \mathrm{nM}$ CRH (Bachem) or forskolin. The phosphodiesterase inhibitor IBMX (5 $\mathrm{mM})$ was added to all stimulation solutions. The supernatants were collected and assayed for cAMP as described previously (Stalla et al. 1989).

\section{Statistical analysis}

Differences were assessed by one-way ANOVA in combination with Scheffé's test, considering $P$ values $<0.05$ as significant.

\section{Results}

TMEFF2 inhibits corticotrope tumor cell proliferation and secretion of ACTH

In the normal human adenohypophysis, TMEFF2 was present in endocrine cells (Fig. 1A, TMEFF2). Double immunohistochemistry revealed that part of the corticotrope population was immunopositive for TMEFF2 (Fig. 1A, TMEFF2+ACTH). Immunohistochemical analysis performed on seven human corticotropinomas revealed variable expression of TMEFF2 (Fig. 1A). Out of seven cases, three had low TMEFF2 immunoreactivity. Four cases did not show expression of TMEFF2.

In accordance with these results, we found that expression of TMEFF2, as determined by RT-PCR, is reduced in ACTH-producing pituitary adenomas from patients with Cushing's syndrome when compared with normal human pituitary tissue (Table 1, TMEFF2/ $\beta$-ACTIN: NP: $3.5 \pm 1.0$, $\mathrm{CUSH}+: 0.8 \pm 0.2$, and $\mathrm{CUSH}++: 1.8)$.

In order to investigate the role of TMEFF2 in AtT20 corticotrope tumor cells, we assessed the effects of TMEFF2 on cell proliferation and production of ACTH. We either silenced expression of TMEFF2 using siRNA or stably overexpressed TMEFF2 in AtT20 cells. As illustrated in Fig. 1B, downregulation of expression of TMEFF2 compared with the level for the scrambled siRNA control led to a significantly higher proliferation rate of the cells (approximately 40\%) $24 \mathrm{~h}$ after stimulation with 10\% FCS. In contrast, AtT20 cells stably transfected with TMEFF2 showed a 35\% decrease in proliferation compared with cells carrying the empty vector (Fig. 1C). Similarly, overexpression of TMEFF2 in AtT20 cells also decreased proliferation measured by the anchorage-independent colony formation assay (Fig. 1D). Although TMEFF2induced inhibition of proliferation of corticotropes would be reflected in a decreased release of ACTH, posttranscriptional compensatory mechanisms cannot be ruled out. To further explore this possibility, we analyzed the involvement of TMEFF2 in production of ACTH.

Published by Bioscientifica Ltd 


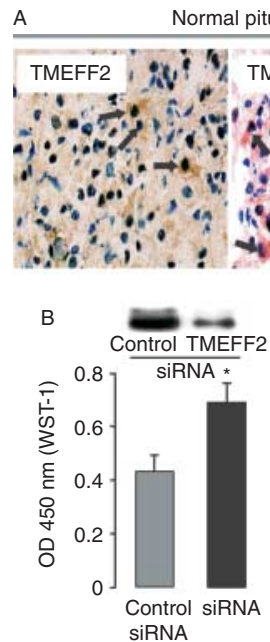

Corticotropinoma

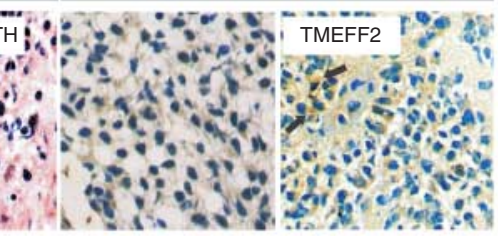

i TMEFF
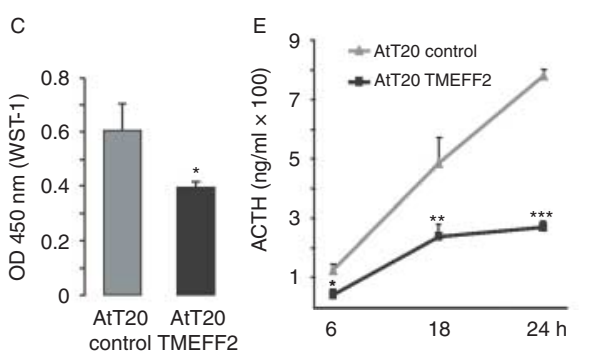

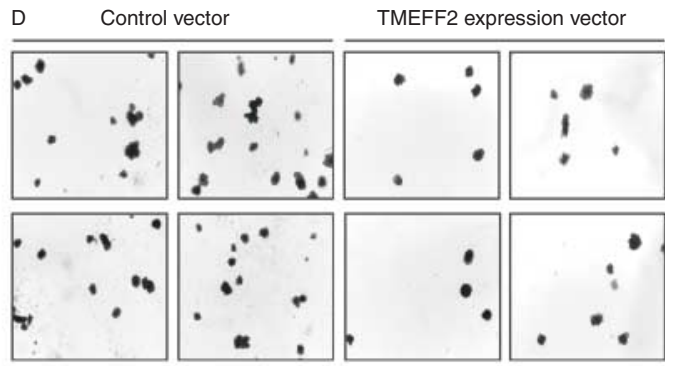

Figure 1

TMEFF2 is expressed in the normal pituitary and inhibits proliferation, colony formation, and production of ACTH in AtT20 cells in vitro. (A) TMEFF2 expression in normal human pituitary was detected by immunohistochemistry (brown color: filled arrows). Colocalization with ACTH-producing cells was assessed by double immunohistochemistry and it is indicated by filled arrows. TMEFF2-negative and -positive corticotropinomas are shown. Nuclei were counterstained with toluidine blue. The images are depicted at a magnification of $40 \times$. Data are representative of at least three different human adult pituitary samples. (B) AtT20 cells were transfected with scramble or TMEFF2 siRNA. Proliferation was measured

AtT20 cells were transiently transfected with TMEFF2 expression vector or its control vector. Twenty-four hours later, the culture medium was changed and supernatants were collected after 6,18 , and $24 \mathrm{~h}$ for determination of ACTH. As expected, TMEFF2 strongly decreased production of ACTH after $24 \mathrm{~h}$ (Fig. 1E).

\section{TMEFF2 inhibits CAMP signaling cascade and decreases transcription of Pomc}

In order to determine the role of TMEFF2 in CRH-stimulated transcription of Pomc, AtT20 cells were cotransfected with either TMEFF2 siRNA or siRNA control together with a Pomc promoter-luciferase reporter plasmid. Transfected cells were incubated in the presence or absence of $100 \mathrm{nM}$ CRH, a physiological stimulus for transcription of Pomc. As expected, CRH stimulated the transcriptional activity of Pomc in all experiments (Fig. 2). TMEFF2 siRNA induced a twofold increase in basal and 3.5-fold increase in CRHinduced activity of the Pomc promoter when compared with control siRNA-transfected cells (Fig. 2A). Conversely, overexpression of TMEFF2 significantly decreased both basal (30\%) and CRH-induced (40\%) promoter activity compared with control cells transfected with the empty vector using the WST-1 assay $24 \mathrm{~h}$ after stimulation with $10 \%$ FCS. (C) Cells stably transfected with full-length TMEFF2 were used to assess proliferation by the WST-1 assay as for (B). An average from three wells per treatment and S.D. from one representative experiment out of three are shown. (D) AtT20 cells transfected with the TMEFF2 or empty vector were seeded in soft agar. After 20 days, the colonies were stained and photographed. (E) Cells stably transfected with TMEFF2 or control vector were incubated in the presence of $10 \%$ FCS for 6,18 , and $24 \mathrm{~h}$. ACTH was measured by RIA in the supernatants. ${ }^{*} P<0.05, * * P<0.01$, and $* * * P<0.001$ compared with the corresponding basal values.

(Fig. 2B). Overexpression of TMEFF2 over time results in a sustained inhibition of the activity of the Pomc promoter under both basal conditions and $\mathrm{CRH}$ stimulation (Supplementary Fig. 1A, see section on supplementary data given at the end of this article). Remarkably, no effect of TMEFF2 was observed on the activity of the promoter of growth hormone in the GH3 pituitary lactosomatotrope tumor cell line (Supplementary Fig. 1B), indicating that TMEFF2 specifically inhibits transcription of Pomc in ACTH-secreting cells.

To elucidate which elements present on the promoter of Pomc, which are involved in induction of Pomc by CRH, are modulated by TMEFF2, we used luciferase reporters for NurRE- and CRE-dependent activity. We found that downregulation of TMEFF2 increases the transcriptional activity of NurRE and CRE in the presence of CRH, whereas overexpression of TMEFF2 decreases the transcriptional activity of NurRE and CRE (Supplementary Fig. 2, see section on supplementary data given at the end of this article and Fig. 2). These results indicate that the inhibition of the promoter of Pomc by TMEFF2 is at least mediated by NUR77/NURR1 and CREB. The fact that different transcription factors are inhibited by TMEFF2 indicates that this transmembrane protein interferes with

Published by Bioscientifica Ltd. 
Table 1 Expression of TMEFF2 quantified by RT-PCR in normal human pituitary tissue and ACTH-producing pituitary adenomas from patients with Cushing's syndrome

\begin{tabular}{|c|c|c|}
\hline Tissue number & Type & TMEFF2 MRNA \\
\hline 1 & NP & +++ \\
\hline 2 & NP & +++ \\
\hline 3 & CUSH & ++ \\
\hline 4 & CUSH & - \\
\hline 5 & CUSH & - \\
\hline 6 & CUSH & + \\
\hline 7 & CUSH & + \\
\hline 8 & CUSH & - \\
\hline 9 & CUSH & + \\
\hline 10 & CUSH & - \\
\hline 11 & CUSH & - \\
\hline 12 & CUSH & - \\
\hline 13 & CUSH & + \\
\hline 14 & CUSH & - \\
\hline
\end{tabular}

NP, normal pituitary; CUSH, Cushing's.

upstream signaling processes at the level of the CRHR1GPCR or its associated signaling cascades.

Then, we assessed the effects of TMEFF2 siRNA on activity of the Pomc by adding stimuli of Pomc other than CRH, like AVP, which signals through Gq or IL6, signaling through STAT. Luciferase analysis of protein lysates derived from AVP- or IL6-treated corticotrope cells showed no changes in promoter activity in the presence of TMEFF2 siRNA compared with control (Supplementary Fig. 3, see section on supplementary data given at the end of this article). This indicates that the effects of TMEFF2 may lead to inhibition of the Pomc promoter only in the presence of GPCR-Gs signaling as neither Gq nor STAT-mediated signaling was affected by TMEFF2.

Then, we examined the cAMP production in AtT20 cells transfected with either control or TMEFF2 siRNA. In the presence of CRH, TMEFF2 siRNA produced a significant increase in the levels of cAMP compared with control siRNA-transfected cells (Fig. 2E), whereas the inhibition of TMEFF2 using siRNA had no significant effect on the basal levels of cAMP. The opposite picture was observed when cells where transfected with TMEFF2 overexpression plasmid and stimulated with forskolin. TMEFF2 decreases levels of cAMP in the presence of forskolin (Fig. 2F). These results indicate that TMEFF2 might regulate cAMP levels either inhibiting AC or stimulating phosphodiesterases. To further characterize the levels of action of TMEFF2 on this pathway, we tested cAMP downstream signaling. In the presence of 8-CPT-cAMP, a lipophilic activator of cAMPdependent protein kinase, TMEFF2 decreases the transcriptional activity of CREB (Fig. 2G). However, cells transfected with PKA catalytic subunits showed no changes in luciferase activity driven by $C R E$-binding sites in the presence of TMEFF2 (Fig. 2H), indicating that TMEFF2 does not influence the catalytic activity of PKA or downstream events.

\section{The activity of TMEFF2 depends on the follistatin domains and TMEFF2 also acts as a secreted protein}

In order to elucidate how TMEFF2 interferes, at the molecular level, with the CRH signaling pathway, we designed five distinct TMEFF2 constructs allowing discrimination between the different domains of TMEFF2 as indicated in the Fig. 3 . The ' $\Delta$ GPD' mutant expresses the extracellular and transmembrane domain but not the intracellular domain carrying the potential $G$ protein domain. The 'sec' (secreted)-TMEFF2 mutant construct contains only the extracellular domain and therefore encodes a secreted protein. No EGF domain is present in the ' $\Delta \mathrm{EGF}$ ' and ' $\Delta \mathrm{EGF} \mathrm{sec}^{\prime}$ mutant constructs; however,

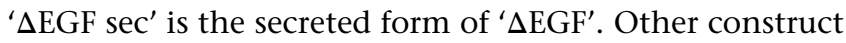
' $\Delta$ Foll- $\Delta \mathrm{EGF}$ ' expresses the transmembrane domain and the intracellular domain with the potential $G$ protein domain only. All five TMEFF2 mutants contained a C-terminal V5/His epitope in frame with TMEFF2-coding sequence in order to monitor for protein-coding capacity.

Upon transient transfection with the CRE-Luciferase plasmid, cells cotransfected with all the different mutants except for the ' $\Delta$ Foll- $\Delta$ EGF' construct had a significantly lower transcriptional activity than cells transfected with the control vector under basal and CRH-stimulated conditions (Fig. 3). The inhibitory effect of these constructs on the transcriptional activity of $C R E$ was similar to the effects of the WT TMEFF2 construct (Fig. 3B, C, D and $\mathrm{E})$. In contrast, when investigating the effects of the construct encoding the transmembrane and intracellular domain alone, ' $\Delta$ Foll- $\Delta E G F$ ' mutant, no effect was observed on the transcriptional activity of CREB (Fig. 3A and F).

Thus, TMEFF2 can act either as a transmembrane or a secreted protein (Lin et al. 2003, Ali \& Knauper 2007) as long as the follistatin domains are present.

\section{TMEFF2 inhibits CRH-induced PI3K and the Ras/Raf pathway}

Emerging evidence indicates that the activity of GPCR is modulated by mechanisms involving different kinases such as PI3k/AKT and raf/ERK1/2 kinase. On the other hand, TMEFF 2 modulates the AKT and ERK1/2 signaling

Published by Bioscientifica Ltd. 
pathways in prostate cell lines (Chen \& Ruiz-Echevarria 2013). Thus, we decided to investigate the role of TMEFF2 in activation of AKT and ERK1/2 in the context of CRH signaling. In these experiments, $\mathrm{CRH}$ stimulation of AtT20 cells resulted in a significant increase in phosphorylation of AKT compared with basal levels in the presence of serum. Interestingly, overexpression of TMEFF2 abolished both basal and CRH-induced phosphorylation of AKT (Fig. 4A). Moreover, we found that TMEFF2 also inhibited
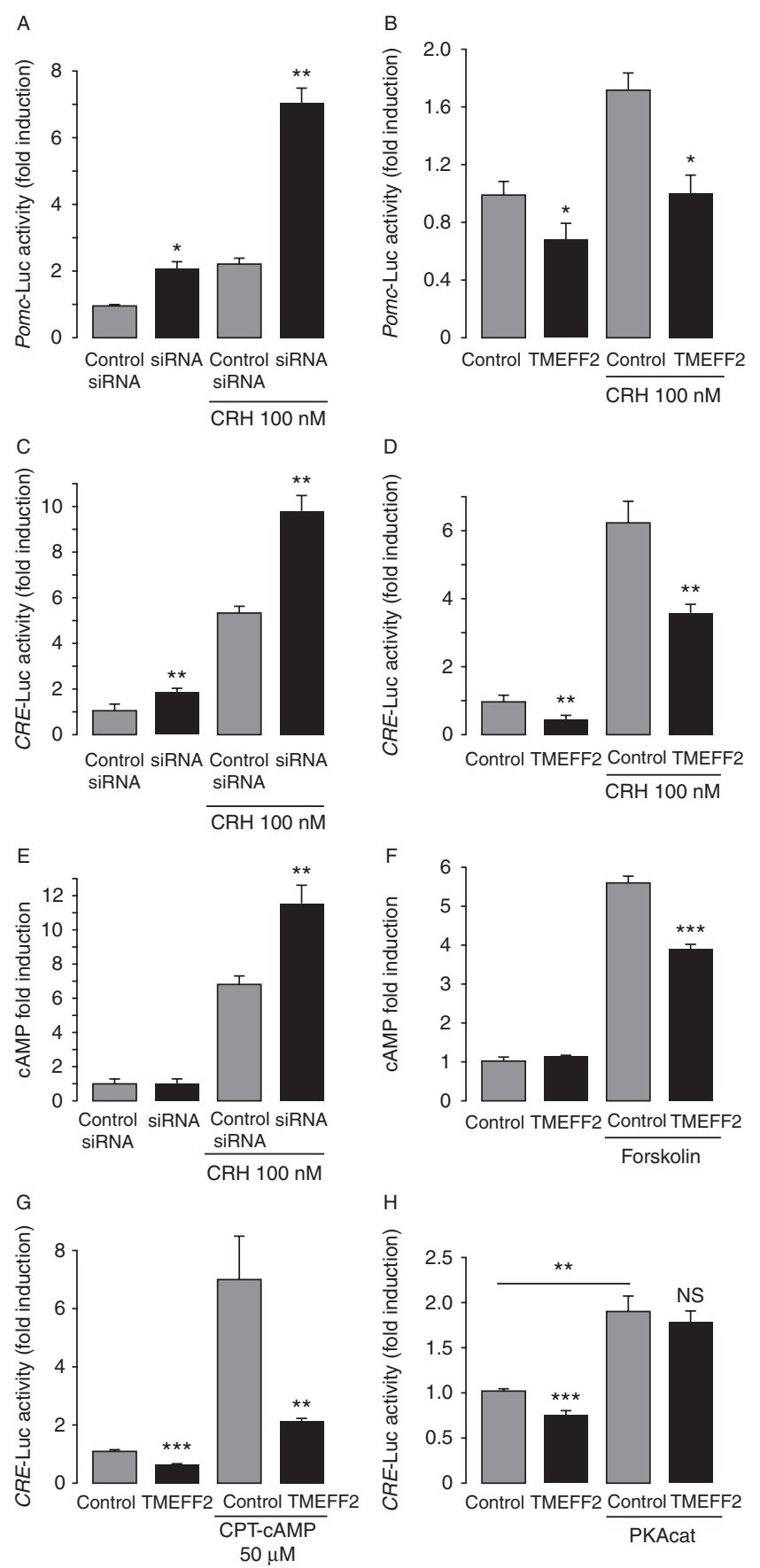

http://jme.endocrinology-journals.org DOI: 10.1530/JME-14-0225 the CRH-induced activation of phosphorylation of ERK1/2 (Fig. 4B). These results indicate an important role for TMEFF2 in the control of the PI3K and MAP kinase pathways in CRH-stimulated corticotrope cells.

\section{TMEFF2 inhibits activin- or BMP4-induced PI3K and Ras/Raf pathway without affecting the transcription of SMAD}

Then, we aimed to ascertain whether modulation of the CRH pathway by TMEFF2 depends on the presence of FCS in the medium. The structural domains of TMEFF2 indicate that it may bind growth factors possibly present in the serum. Besides that, the secreted form of TMEFF2 is sufficient to inhibit the transcriptional activity of CREB.

In order to elucidate the role of growth factors in the inhibitory effect of TMEFF2 on the transcriptional activity of CREB, we treated TMEFF2-transfected AtT20 cells with or without $10 \%$ serum. As shown in Fig. 5A, serum substantially increased the inhibition of luciferase activity driven by $C R E$, indicating that serum components, probably growth factors, play an important role in the inhibitory function of TMEFF2.

Growth factors such as activin and BMP4 were shown to inhibit the expression of Pomc in corticotrope cells involving SMAD-dependent signals. However, activin and BMP4 transmit signals also through SMAD-independent pathways, including the PI3K and ERK1/2 signaling pathways. Several reports have included evidence that Akt and Erk1/2 upregulate the expression of Pomc. As TMEFF2 inhibits CRH-induced phosphorylation of AKT and ERK1/2, we decided to investigate the role of TMEFF2 in the activin and BMP4 signaling pathways.

AtT20 cells were transfected with TMEFF2 or empty vector and stimulated with activin or BMP4 at different time

\section{Figure 2}

TMEFF2 inhibits $\mathrm{CRH}$-induced stimulation of CAMP, CRE, and transcription of Pomc. (A and C) AtT20 cells were cotransfected with either TMEFF2 siRNA or control siRNA and Pomc-Luc (A) or CRE-Luc (C). (B and D) AtT20 cells were cotransfected with either the TMEFF2-encoding plasmid or the empty vector and Pomc-Luc (B) or CRE-LuC (D). (A, B, C and D) After 48 h, cells were treated with $C R H$. (E and F) AtT20 cells were transfected with either TMEFF2 siRNA or control siRNA (E) or with the TMEFF2 expression vector or empty plasmid (F) and treated with $\mathrm{CRH}$ or forskolin for $1 \mathrm{~h}$ respectively. Intracellular CAMP levels were measured as described in the 'Materials and methods'. (G and H) AtT20 cells were cotransfected with the TMEFF2 expression vector or empty plasmid and CRE-Luc. Cells were treated as indicated in the figure (G). AtT20 cells were cotransfected with the PKA catalytic subunit expression plasmid or control vector $(H)$. Results are expressed as the fold induction relative to the basal value, arbitrarily taken as 1 . Values indicate the mean \pm s.D. of triplicates from one representative experiment $(n=3)$. ${ }^{\star} P<0.05, * * P<0.01$, and $* * * P<0.001$ compared with the corresponding control or basal value. 

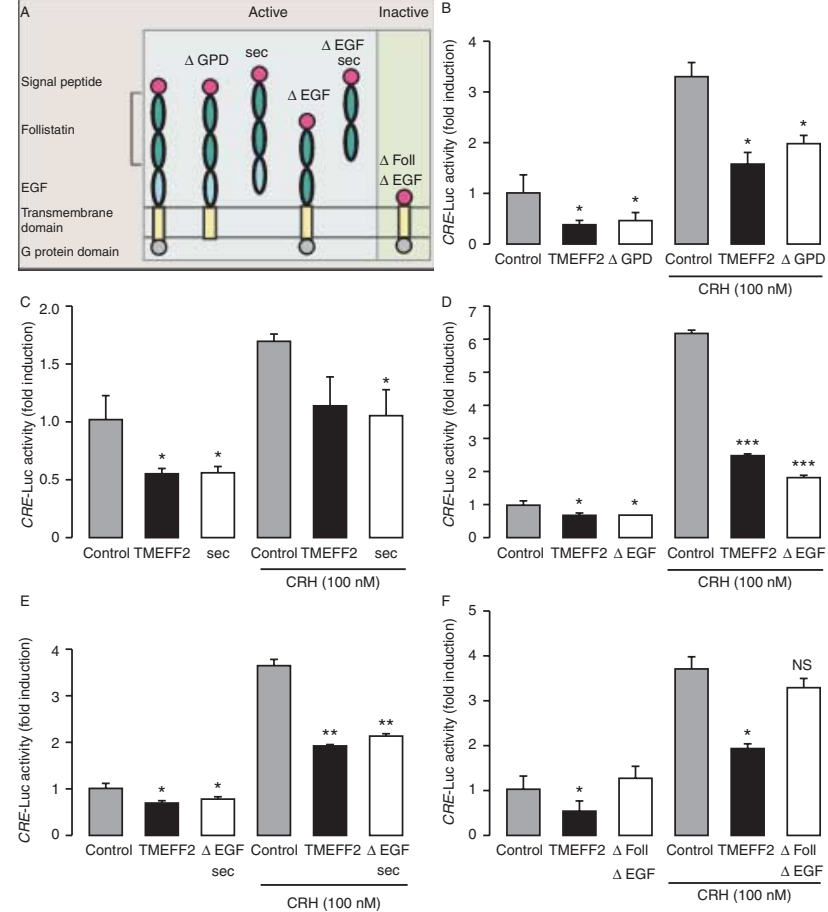

Figure 3

Follistatin domains are essential for the inhibition of transcription of CRELuc. (A) Deletion mutants of the TMEFF2 protein. (B, C, D, E and F) AtT20 cells were cotransfected with the control plasmid, full-length TMEFF2 expression vector, and different TMEFF2 mutants as indicated in the figures and CRE-Luc. Cells were treated with $100 \mathrm{nM} \mathrm{CRH}$ for $6 \mathrm{~h}$. Results are expressed as the fold induction relative to the basal value, arbitrarily taken as 1 . Values indicate the mean \pm s.D. of triplicates from one representative experiment $(n=3) .{ }^{*} P<0.05, * * P<0.01$, and $* * * P<0.001$, ns, not statistically significant compared with the corresponding control value.

points. Cell lysates were then analyzed. As shown in Fig. 5B, overexpression of TMEFF2 decreased the phosphorylation of AKT at Ser ${ }^{473}$ without affecting the expression of total AKT, indicating either inhibition of mTOR or activation of phosphatase by TMEFF2. In addition, TMEFF2 also inhibited activin- or BMP4-induced $\mathrm{P} 42 / 44$ phosphorylation of ERK1/2 (Fig. 5C). Furthermore, overexpression of TMEFF2 blocked basal and activin- or BMP4-induced activation of transcription of Gal4-Elk1, a well-characterized reporter of ERK1/2-dependent activity (Supplementary Fig. 4A and B, see section on supplementary data given at the end of this article). These results indicate that TMEFF2, in fact, diminishes ERK1/2 and the activation of PI3K/AKT induced by activin and BMP4.

In another set of experiments, AtT20 cells were cotransfected with a reporter of SMAD-dependent transcriptional activity and the TMEFF2 expression vector in the presence of activin. No changes were observed in cells transfected with TMEFF2 compared with controls, indicating that TMEFF2 does not interfere with activininduced transcription of SMAD (Supplementary Fig. 4C). The same results were obtained by stimulating cells with BMP4 (data not shown).

\section{TMEFF2 increases activin-induced and BMP4-induced inhibition of POMC transcriptional activity}

Then, we tested the role of TMEFF2 in activin- and BMP4induced inhibition of Pomc. Transfected cells were incubated in the presence or absence of activin (Fig. 6A) or BMP4 (Fig. 6B). Activin and BMP4 inhibited the transcription of Pomc (Fig. 6), as reported previously. As expected, TMEFF2 inhibited the transcription of Pomc and, in the presence of activin or BMP4, TMEFF2 reduced the expression of Pomc to a further extent (Fig. 6) beyond the inhibition caused by activin/BMP4 or TMEFF2 alone.

Based on these results, we present a model for TMEFF2-induced transcriptional inhibition of Pomc in which TMEFF2 acts at different levels inhibiting phosphorylation of ERK1/2 and AKT and cAMP signaling (Fig. 7). When activin or BMP4 are present, repression of transcription of Pomc by SMAD may be reinforced by inhibition by TMEFF2 of the activation of ERK1/2 and AKT (Fig. 7).

\section{Discussion}

The effects of CRH on corticotrope cells in the pituitary gland are mediated by G protein-coupled CRHR1. Stimulation of the receptor by CRH activates a Gs-protein that in turn activates the AC, resulting in the conversion of ATP to

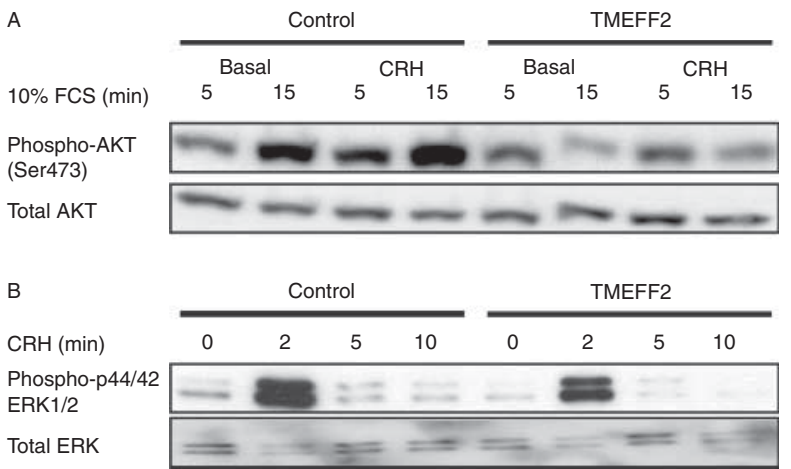

\section{Figure 4}

TMEFF2 inhibits CRH-induced phosphorylation of AKT (A) and ERK1/2 (B). AtT20 cells were transfected with either the full-length TMEFF2 expression vector or control plasmid and stimulated with $\mathrm{CRH}$ at the indicated time points. Cell lysates were analyzed by WB as described in the 'Materials and methods'. Results from one representative experiment are shown $(n=3)$.

Published by Bioscientifica Ltd 


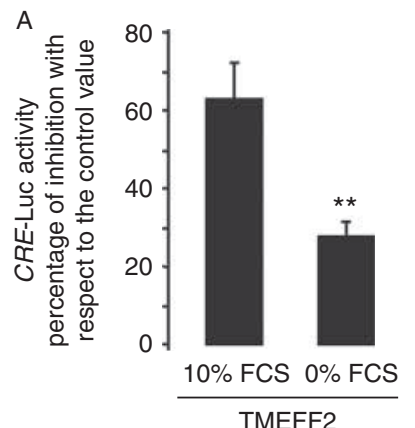

B

Activin (min)

Phospho-AKT (Ser473)

Total AKT

Phospho-p44/42 ERK1/2

Total ERK

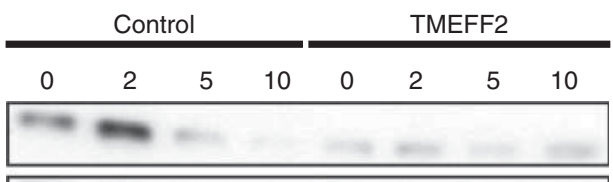

C

BMP4 (min)

Phospho-AKT

(Ser473)

Total AKT

Phospho-p44/42 ERK1/2

Total ERK
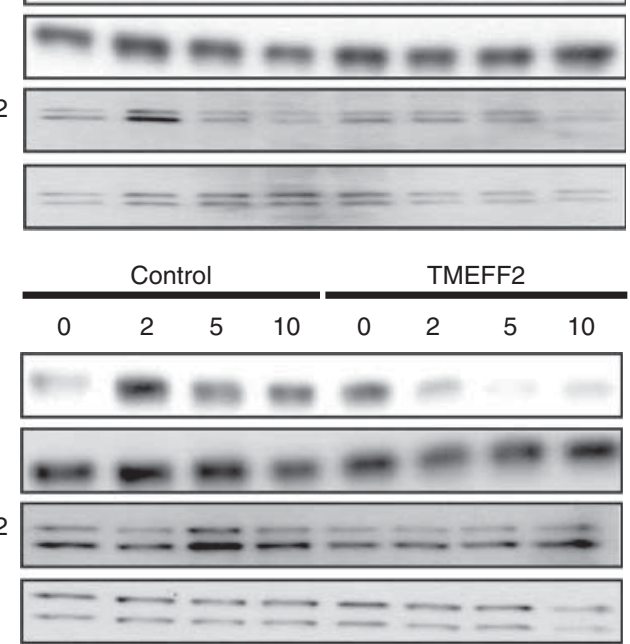

\section{Figure 5}

TMEFF2 inhibits activin- and BMP4-induced phosphorylation of AKT and ERK1/2. (A) TMEFF2-induced inhibition of transcription of CREB depends on serum components. AtT20 cells were cotransfected with either the fulllength TMEFF2 expression vector or control plasmid and CRE-Luc. Cells were treated with 0 or $10 \%$ FCS for $6 \mathrm{~h}$. Results are expressed as percentage inhibition with respect to the control values. Values indicate the mean \pm s.D. of triplicates from one representative experiment $(n=3) . * * P<0.01$. (B and C) AtT20 cells were transfected with either the full-length TMEFF2 expression vector or control plasmid and stimulated with activin or BMP4 at the indicated time points. Cell lysates were analyzed by western blotting. One representative experiment out of three independent experiments is shown.

cAMP. cAMP activates PKA, leading to the transcription of Pomc and synthesis of ACTH. Our results demonstrate for the first time, to our knowledge, that TMEFF2 is expressed in pituitary corticotrope cells and that expression of TMEFF2 inhibits the effects of CRH on pituitary corticotrope tumor cells, decreasing cAMP and blocking transcription of Pomc. These results indicate that TMEFF2 acts as an inhibitor of the CRH signal transduction pathway. Conversely and confirming these results, the downregulation of TMEFF2 using siRNA results in the activation of CRHR1 signaling. Partial inhibition of CRH-induced activity of Pomc by TMEFF2 may be due to low expression levels or incomplete trafficking and insertion of the ectopic protein into the plasma membrane.

In corticotropes, CRH activates CREB transcription factor and induces NUR77 and NURR1, which bind NurRE and NBRE sites to promote transcriptional activity. We found that TMEFF2 inhibited Pomc-, CREB-, and Nur-dependent activities in AtT20 cells under basal or CRH-stimulated conditions. Our results do not exclude the possibility that other factors, like PITx/TPit, may also be involved in repression of Pomc by TMEFF2, as repression of transcription of Pomc by interference with PITx/TPIT has also been described (Nudi et al. 2005). Importantly, we found that TMEFF2 affected not only transcriptional
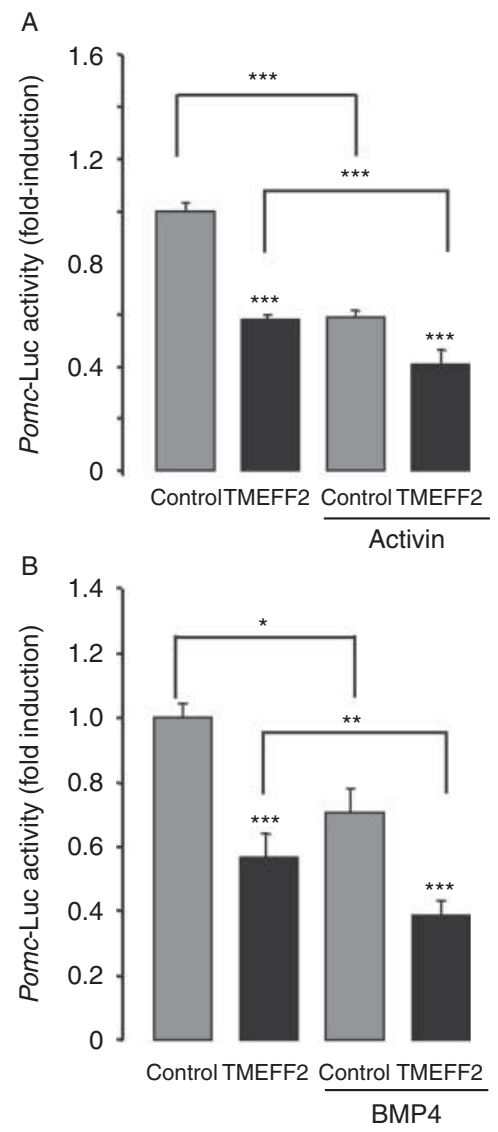

Figure 6

TMEFF2 enhances inhibition of POMC transcriptional activity induced by activin or BMP4. AtT20 cells were cotransfected with either the full-length TMEFF2 expression vector or control plasmid and Pomc-Luc. Cells were stimulated with activin (A) or BMP4 (B). Results are expressed as the fold induction relative to the basal value, arbitrarily taken as 1 . Values indicate the mean \pm s.D. of triplicates from one representative experiment $(n=3)$. ${ }^{\star} P<0.05,{ }^{* *} P<0.01$ and $* * * P<0.001$ compared with the corresponding control or basal value.

Published by Bioscientifica Ltd. 


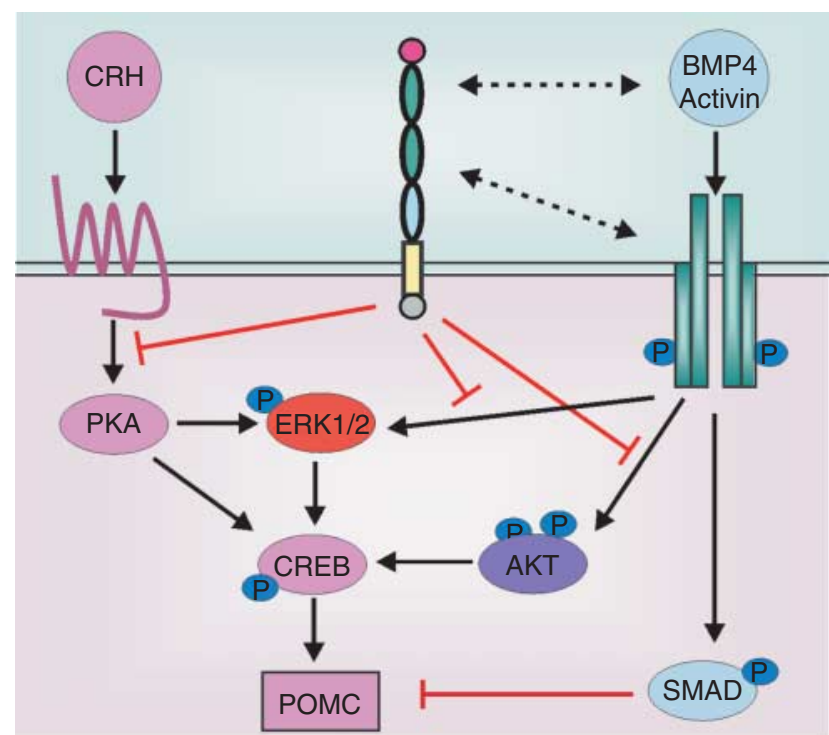

Figure 7

Model of TMEFF2 inhibition of POMC. TMEFF2 inhibits the CRH signaling pathway leading to reduced transcription of Pomc. TMEFF2 acts at different levels, also inhibiting phosphorylation of AKT and ERK1/2, and thus enhancing inhibition of Pomc triggered by stimulation with BMP4 or activin. TMEFF2 may interact with BMP4/activin or their receptors in order to inhibit non-canonical pathways.

activity of Pomc but also consequently endogenous production of ACTH in AtT20 cells. Moreover, TMEFF2 colocalizes with ACTH-producing cells in the normal human adenohypophysis and its effect on these cells seems to be specific as TMEFF2 does not affect the transcriptional activity of $\mathrm{GH}$, a hormone produced by lactosomatotrope cells inside the pituitary. The role of TMEFF2 in production of ACTH by normal murine pituitary cells and human corticotropinomas remains yet to be elucidated. However, the significance of the inhibition of Pomc/ACTH, reported in this study, with respect to the potential therapeutic use of TMEFF2 or factors stimulating the expression and activity of TMEFF2 for the treatment of Cushing's syndrome is emphasized by the low levels of expression of TMEFF2 in human corticotropinomas compared with normal tissue. In this respect, it has been reported that downregulation of TMEFF2 in androgen-independent prostate cancer xenografts, as well as growth inhibition induced by ectopic expression of TMEFF2 in androgen-independent prostate cancer cell lines (Gery et al. 2002). Conversely, however, lower levels of TMEFF 2 are found in multiple cancer tissues, especially in the malignant brain and colorectal samples, when compared with normal tissue (Horie et al. 2000). These findings together with our results indicate a possible tissuedependent function of TMEFF2 in different human tumors.
Our results further indicated that the inhibition of TMEFF2 by siRNA in pituitary corticotrope tumor cells increases cell proliferation, indicating that this protein exerts antiproliferative effects on these cells. Thus, in agreement with the findings of Gery et al. (2002) in prostate cancer cells, we demonstrated, in this study, that overexpression of TMEFF2 leads to the inhibition of proliferation of tumoral pituitary cells. Moreover, the fact that expression of TMEFF2 is reduced in Cushing's adenomas when compared with human normal pituitary may indicate that TMEFF2 could play a role in preventing development of tumors. In this study, we further extended our analysis of the biological effects of TMEFF 2 on AtT20 cells focusing on previously unexplored properties, namely anchorage-independent colony formation in soft agar, a hallmark of malignant transformation. Overexpression of TMEFF2 markedly decreased the ability of AtT20 cells to form colonies in soft agar. Taken together, these results indicate that TMEFF2 might be an interesting therapeutic option for inhibiting production of ACTH and tumor growth in Cushing's syndrome.

In the pituitary cell line AtT20, TMEFF2 specifically inhibited GPCR-Gs signaling without affecting signals mediated by $\mathrm{Gq}$ or STAT. In colon cancer cell lines, overexpression of TMEFF2 leads to upregulation of STAT1 and suppression of growth (Elahi et al. 2008). These opposing effects of TMEFF2 acting or not on activation of STAT may reflect the use of distinct signaling cascades by TMEFF2 in different tissues probably modulating different functions, e.g., hormone production versus cell growth.

Interestingly, while the full-length TMEFF2 protein, TMEFF2 lacking the G protein domain, the secreted TMEFF2 ectodomain, and TMEFF2 isoforms without the EGF domains inhibited basal and CRH-induced CREB transcriptional activity, the intracellular and transmembrane isoform had no effect on CRE-Luc activity. Therefore, the follistatin domain of TMEFF2 is essential for the biological activity of the protein in the CRH signaling pathway in AtT20 cells.

In this study, we report for the first time, to our knowledge, that TMEFF2 inhibits GPCR-Gs signaling by regulating the activity of the PKA regulatory subunit and/or the AC. In epithelial prostate cells (Chen \& Ruiz-Echevarria 2013), it has been observed that TMEFF2 modulates the activity of the Ras/ERK1/2 and PI3K/AKT signaling pathways. Herein, we explored the effect of full-length TMEFF2 on these two signaling pathways in the corticotrope cell line, AtT20. At the molecular level, TMEFF2 modulated activation of ERK1/2 and AKT by inhibiting the

Published by Bioscientifica Ltd. 
phosphorylation induced by $\mathrm{CRH}$, activin, or BMP4. Ras/ERK1/2 and PI3K/AKT play critical roles in control of cell survival, proliferation, and differentiation, and both pathways may function together to promote tumorigenesis (Grant 2008, Cakir \& Grossman 2009). Our results describing the TMEFF2-mediated inhibition of phosphorylation of AKT and ERK1/2 are consistent with the hypothesis of TMEFF2 acting as a tumor suppressor by blocking phosphorylation of AKT and ERK1/2 and consequently proliferation and colony formation in tumor corticotrope cells. Crosstalk of different signaling transduction pathways plays a central adaptive role in the regulation of cell function. Emerging evidence indicates that the activity of GPCR is modulated by mechanisms involving different kinases such as PI3k/AKT and ERK1/2 kinases. Thus, the following observations can be made: AKT is capable of phosphorylating and activating CREB (Peltier et al. 2007), the catalytic subunit of PKA is an excellent substrate for PDK1 (Cheng et al. 1998), and ERK1/2 can activate CREB (Adams et al. 2000, Steelman etal. 2008) and Nurr (Kovalovsky etal. 2002). Moreover, ERK1/2 inhibits long PDE4 isoforms and activates short ones, which in turn regulates basal levels of cAMP (Cheng et al. 1998). Thus, in corticotrope cells, Ras/Erk1/2, PI3K/Akt, and CRH signaling pathways in the presence of TMEFF2 may crosstalk, affecting the outcome of the response and hence blocking further steps in the $\mathrm{CRH}$ transduction pathway. Although the molecular function of TMEFF2 is largely unknown, its structural domains indicate that it may act as an inhibitor of growth factors. In other proteins containing follistatin domains, it has been shown that follistatin binds and inhibits the function of growth factors, like activin (Patthy \& Nikolics 1993, Kupprion et al. 1998, Patel 1998) and platelet-derived growth factor (PDGF; Lin et al. 2011). Recently, PDGFAA has been described as a growth factor that interacts with a recombinant form of the ectodomain of TMEFF2 to suppress PDGF receptor signaling and interferes with PDGFAA-stimulated proliferation of NR6 fibroblasts (Lin et al. 2011). Pdgfa mRNA was not detected in AtT20 cells; nevertheless, $P d g f b$ and the receptor $\alpha$ are expressed in corticotrope cells (Kowarik et al. 2010). PDGFA and PDGFB (A and B chains) transcripts as well as the receptor mRNAs were found in rat pituitary (Kowarik et al. 2010). However, little is known about the functional importance of this growth factor in this gland. PDGF receptor binding activates several signaling systems such as PI3K and Ras. Thus, it might be that a PDGF isoform acts as a functional ligand of TMEFF2. Thus, it could be possible that TMEFF2 regulates PDGF signaling in pituitary corticotropes. Future experiments, in particular coimmunoprecipitation experiments, are required to shed light on this particular issue. Identification of partners interacting with TMEFF2 in corticotrope cells would facilitate the study of the role of TMEFF2 in these cells and the potential development of drugs that stimulate its activity.

In summary, our results indicate a potential therapeutic use of TMEFF2 or factors that stimulate activity of TMEFF2 for the treatment of corticotrope tumors in order to reduce secretion of ACTH and proliferation.

\section{Supplementary data}

This is linked to the online version of the paper at http://dx.doi.org/10.1530/ JME-14-0225.

\section{Declaration of interest}

M L: the Max Planck Institute of Psychiatry has a pending patent related to this work.

\section{Funding}

This research did not receive any specific grant from any funding agency in the public, commercial or not-for-profit sector.

\section{Author contribution statement}

$\mathrm{M} \mathrm{L}$ and $\mathrm{G} \mathrm{KS}$ conceived and designed the experiments and wrote the manuscript. B W and J S performed the experiments. M L analyzed the data.

\section{Acknowledgements}

The authors would like to thank $D$ Refojo for critical reading of the manuscript. The authors would like to thank Affectis Pharmaceuticals, Germany for the plasmids.

\section{References}

Adams JP, Roberson ED, English JD, Selcher JC \& Sweatt JD 2000 MAPK regulation of gene expression in the central nervous system. Acta Neurobiologiae Experimentalis $60377-394$.

Ali N \& Knauper V 2007 Phorbol ester-induced shedding of the prostate cancer marker transmembrane protein with epidermal growth factor and two follistatin motifs 2 is mediated by the disintegrin and metalloproteinase-17. Journal of Biological Chemistry 282 37378-37388. (doi:10.1074/jbc.M702170200)

Bale TL \& Vale WW 2004 CRF and CRF receptors: role in stress responsivity and other behaviors. Annual Review of Pharmacology and Toxicology 44 525-557. (doi:10.1146/annurev.pharmtox.44.101802.121410)

Birnbaumer M 2000 Vasopressin receptors. Trends in Endocrinology and Metabolism 11 406-410. (doi:10.1016/S1043-2760(00)00304-0)

Boutillier AL, Monnier D, Lorang D, Lundblad JR, Roberts JL \& Loeffler JP 1995 Corticotropin-releasing hormone stimulates proopiomelanocortin transcription by cFos-dependent and -independent pathways: characterization of an AP1 site in exon 1. Molecular Endocrinology 9 745-755. (doi:10.1210/mend.9.6.8592520)

Published by Bioscientifica Ltd. 
Boutillier AL, Gaiddon C, Lorang D, Roberts JL \& Loeffler JP 1998 Transcriptional activation of the proopiomelanocortin gene by cyclic AMP-responsive element binding protein. Pituitary $133-43$. (doi:10.1023/A:1009966808106)

Cakir M \& Grossman AB 2009 Targeting MAPK (Ras/ERK) and PI3K/Akt pathways in pituitary tumorigenesis. Expert Opinion on Therapeutic Targets 13 1121-1134. (doi:10.1517/14728220903170675)

Castillo V, Giacomini D, Paez-Pereda M, Stalla J, Labeur M, Theodoropoulou M, Holsboer F, Grossman AB, Stalla GK \& Arzt E 2006 Retinoic acid as a novel medical therapy for Cushing's disease in dogs. Endocrinology 147 4438-4444. (doi:10.1210/en.2006-0414)

Chen X \& Ruiz-Echevarria MJ 2013 TMEFF2 modulates the AKT and ERK signaling pathways. International Journal of Biochemistry and Molecular Biology 4 83-94.

Cheng X, Ma Y, Moore M, Hemmings BA \& Taylor SS 1998 Phosphorylation and activation of cAMP-dependent protein kinase by phosphoinositide-dependent protein kinase. PNAS 95 9849-9854. (doi:10.1073/pnas.95.17.9849)

Chesnokova V \& Melmed S 2002 Minireview: Neuro-immuno-endocrine modulation of the hypothalamic-pituitary-adrenal (HPA) axis by gp130 signaling molecules. Endocrinology 143 1571-1574. (doi:10.1210/endo.143.5.8861)

Dahia PL \& Grossman AB 1999 The molecular pathogenesis of corticotroph tumors. Endocrine Reviews 20 136-155. (doi:10.1210/edrv.20.2.0358)

Elahi A, Zhang L, Yeatman TJ, Gery S, Sebti S \& Shibata D 2008 HPP1-mediated tumor suppression requires activation of STAT1 pathways. International Journal of Cancer 122 1567-1572. (doi:10.1002/ ijc.23202)

Gagner JP \& Drouin J 1987 Tissue-specific regulation of pituitary proopiomelanocortin gene transcription by corticotropin-releasing hormone, 3',5'-cyclic adenosine monophosphate, and glucocorticoids. Molecular Endocrinology 1 677-682. (doi:10.1210/mend-1-10-677)

Gery S, Sawyers CL, Agus DB, Said JW \& Koeffler HP 2002 TMEFF2 is an androgen-regulated gene exhibiting antiproliferative effects in prostate cancer cells. Oncogene 21 4739-4746. (doi:10.1038/sj.onc. 1205142)

Giacomini D, Paez-Pereda M, Theodoropoulou M, Labeur M, Refojo D, Gerez J, Chervin A, Berner S, Losa M, Buchfelder M et al. 2006 Bone morphogenetic protein- 4 inhibits corticotroph tumor cells: involvement in the retinoic acid inhibitory action. Endocrinology 147 247-256. (doi:10.1210/en.2005-0958)

Glynne-Jones E, Harper ME, Seery LT, James R, Anglin I, Morgan HE, Taylor KM, Gee JM \& Nicholson RI 2001 TENB2, a proteoglycan identified in prostate cancer that is associated with disease progression and androgen independence. International Journal of Cancer 94 178-184. (doi:10.1002/ijc.1450)

Grammatopoulos DK \& Chrousos GP 2002 Functional characteristics of CRH receptors and potential clinical applications of CRH-receptor antagonists. Trends in Endocrinology and Metabolism 13 436-444. (doi:10.1016/S1043-2760(02)00670-7)

Grant S 2008 Cotargeting survival signaling pathways in cancer. Journal of Clinical Investigation 118 3003-3006. (doi:10.1172/JCI36898E1)

Horie M, Mitsumoto Y, Kyushiki H, Kanemoto N, Watanabe A, Taniguchi Y, Nishino N, Okamoto T, Kondo M, Mori T et al. 2000 Identification and characterization of TMEFF2, a novel survival factor for hippocampal and mesencephalic neurons. Genomics 67 146-152. (doi:10.1006/geno. 2000.6228)

Kanemoto N, Horie M, Omori K, Nishino N, Kondo M, Noguchi K \& Tanigami A 2001 Expression of TMEFF1 mRNA in the mouse central nervous system: precise examination and comparative studies of TMEFF1 and TMEFF2. Brain Research. Molecular Brain Research 86 48-55. (doi:10.1016/S0169-328X(00)00257-6)

Kovalovsky D, Refojo D, Liberman AC, Hochbaum D, Pereda MP, Coso OA, Stalla GK, Holsboer F \& Arzt E 2002 Activation and induction of NUR77/NURR1 in corticotrophs by CRH/cAMP: involvement of calcium, protein kinase A, and MAPK pathways. Molecular Endocrinology 16 1638-1651. (doi:10.1210/mend.16.7.0863)

Kowarik M, Onofri C, Colaco T, Stalla GK \& Renner U 2010 Platelet-derived growth factor (PDGF) and PDGF receptor expression and function in folliculostellate pituitary cells. Experimental and Clinical Endocrinology \& Diabetes 118 113-120. (doi:10.1055/s-0029-1202832)

Kupprion C, Motamed K \& Sage EH 1998 SPARC (BM-40, osteonectin) inhibits the mitogenic effect of vascular endothelial growth factor on microvascular endothelial cells. Journal of Biological Chemistry 273 29635-29640. (doi:10.1074/jbc.273.45.29635)

Leung CK, Paterson JA, Imai Y \& Shiu RP 1982 Transplantation of ACTHsecreting pituitary tumor cells in athymic nude mice. Virchows Archiv. A, Pathological Anatomy and Histology 396 303-312. (doi:10.1007/ BF00431389)

Liang G, Robertson KD, Talmadge C, Sumegi J \& Jones PA 2000 The gene for a novel transmembrane protein containing epidermal growth factor and follistatin domains is frequently hypermethylated in human tumor cells. Cancer Research 60 4907-4912.

Lin H, Wada K, Yonezawa M, Shinoki K, Akamatsu T, Tsukui T \& Sakamoto C 2003 Tomoregulin ectodomain shedding by proinflammatory cytokines. Life Sciences 73 1617-1627. (doi:10.1016/S00243205(03)00514-9)

Lin K, Taylor JR Jr, Wu TD, Gutierrez J, Elliott JM, Vernes JM, Koeppen $\mathrm{H}$ Phillips HS, de Sauvage FJ \& Meng YG 2011 TMEFF2 is a PDGF-AA binding protein with methylation-associated gene silencing in multiple cancer types including glioma. PLOS ONE 6 e18608. (doi:10.1371/journal.pone.0018608)

Liu B, Mortrud M \& Low MJ 1995 DNA elements with AT-rich core sequences direct pituitary cell-specific expression of the pro-opiomelanocortin gene in transgenic mice. Biochemical Journal 312 827-832.

Maira M, Martens C, Philips A \& Drouin J 1999 Heterodimerization between members of the Nur subfamily of orphan nuclear receptors as a novel mechanism for gene activation. Molecular and Cellular Biology 19 7549-7557.

Maurer RA 1989 Both isoforms of the cAMP-dependent protein kinase catalytic subunit can activate transcription of the prolactin gene. Journal of Biological Chemistry 264 6870-6873.

Murphy EP \& Conneely OM 1997 Neuroendocrine regulation of the hypothalamic pituitary adrenal axis by the nurr $1 /$ nur77 subfamily of nuclear receptors. Molecular Endocrinology 11 39-47. (doi:10.1210/ mend.11.1.9874)

Nudi M, Ouimette JF \& Drouin J 2005 Bone morphogenic protein (Smad)mediated repression of proopiomelanocortin transcription by interference with Pitx/Tpit activity. Molecular Endocrinology 19 1329-1342. (doi:10.1210/me.2004-0425)

Orth DN 1995 Cushing's syndrome. New England Journal of Medicine 332 791-803. (doi:10.1056/NEJM199503233321207)

Paez-Pereda M, Ledda MF, Goldberg V, Chervin A, Carrizo G, Molina H, Muller A, Renner U, Podhajcer O, Arzt E et al. 2000 High levels of matrix metalloproteinases regulate proliferation and hormone secretion in pituitary cells. Journal of Clinical Endocrinology and Metabolism 85 263-269. (doi:10.1210/jcem.85.1.6248)

Paez-Pereda M, Kovalovsky D, Hopfner U, Theodoropoulou M, Pagotto U, Uhl E, Losa M, Stalla J, Grubler Y, Missale C et al. 2001 Retinoic acid prevents experimental Cushing syndrome. Journal of Clinical Investigation 108 1123-1131. (doi:10.1172/JCI11098)

Patel K 1998 Follistatin. International Journal of Biochemistry \& Cell Biology 30 1087-1093. (doi:10.1016/\$1357-2725(98)00064-8)

Patthy L \& Nikolics K 1993 Functions of agrin and agrin-related proteins. Trends in Neurosciences 16 76-81. (doi:10.1016/0166-2236 (93)90021-D)

Pecori Giraldi F, Ambrogio AG, Andrioli M, Sanguin F, Karamouzis I, Corsello SM, Scaroni C, Arvat E, Pontecorvi A \& Cavagnini F 2012 Potential role for retinoic acid in patients with Cushing's disease. The Journal of Clinical Endocrinology \& Metabolism 97 3577-3583. (doi:10.1210/jc.2012-2328) http://jme.endocrinology-journals.org

DOI: 10.1530/JME-14-0225
() 2015 Society for Endocrinology Printed in Great Britain 
Peltier J, O'Neill A \& Schaffer DV 2007 PI3K/Akt and CREB regulate adult neural hippocampal progenitor proliferation and differentiation. Developmental Neurobiology 67 1348-1361. (doi:10.1002/ dneu.20506)

Perrin MH \& Vale WW 1999 Corticotropin releasing factor receptors and their ligand family. Annals of the New York Academy of Sciences $\mathbf{8 8 5}$ 312-328. (doi:10.1111/j.1749-6632.1999.tb08687.x)

Philips A, Lesage S, Gingras R, Maira MH, Gauthier Y, Hugo P \& Drouin J 1997 Novel dimeric Nur77 signaling mechanism in endocrine and lymphoid cells. Molecular and Cellular Biology 17 5946-5951.

Reul JM \& Holsboer F 2002 Corticotropin-releasing factor receptors 1 and 2 in anxiety and depression. Current Opinion in Pharmacology 2 23-33. (doi:10.1016/S1471-4892(01)00117-5)

Stalla GK, Stalla J, von Werder K, Müller OA, Gerzer R, Höllt V \& Jakobs KH 1989 Nitroimidazole derivatives inhibit anterior pituitary cell function apparently by a direct effect on the catalytic subunit of the adenylate cyclase holoenzyme. Endocrinology 125 699-706. (doi:10.1210/endo-125-2-699)

Steelman LS, Abrams SL, Whelan J, Bertrand FE, Ludwig DE, Basecke J, Libra M, Stivala F, Milella M, Tafuri A et al. 2008 Contributions of the Raf/MEK/ERK, PI3K/PTEN/Akt/mTOR and Jak/STAT pathways to leukemia. Leukemia 22 686-707. (doi:10.1038/leu.2008.26)
Therrien M \& Drouin J 1991 Pituitary pro-opiomelanocortin gene expression requires synergistic interactions of several regulatory elements. Molecular and Cellular Biology 11 3492-3503. (doi:10.1128/ MCB.11.7.3492)

Tse A \& Lee AK 1998 Arginine vasopressin triggers intracellular calcium release, a calcium-activated potassium current and exocytosis in identified rat corticotropes. Endocrinology 139 2246-2252. (doi:10.1210/endo.139.5.5999)

Uchida T, Wada K, Akamatsu T, Yonezawa M, Noguchi H, Mizoguchi A, Kasuga M \& Sakamoto C 1999 A novel epidermal growth factor-like molecule containing two follistatin modules stimulates tyrosine phosphorylation of erbB-4 in MKN28 gastric cancer cells. Biochemical and Biophysical Research Communications 266 593-602. (doi:10.1006/ bbrc.1999.1873)

Vossler MR, Yao H, York RD, Pan MG, Rim CS \& Stork PJ 1997 cAMP activates MAP kinase and Elk-1 through a B-Raf- and Rap1dependent pathway. Cell 89 73-82. (doi:10.1016/S0092-8674(00) 80184-1)

YoungJ, Biden KG, Simms LA, Huggard P, Karamatic R, Eyre HJ, Sutherland GR, Herath N, Barker M, Anderson GJ et al. 2001 HPP1: a transmembrane protein-encoding gene commonly methylated in colorectal polyps and cancers. PNAS 98 265-270. (doi:10.1073/pnas.98.1.265)

Received in final form 20 November 2014

Accepted 28 November 2014
(C) 2015 Society for Endocrinology Printed in Great Britain
Published by Bioscientifica Ltd. 